\title{
Robust Stability of Singularly Perturbed Systems with Delays*
}

\author{
Yang Yang and Yuan Wang ${ }^{1}$
}

\begin{abstract}
We consider in this work stability of singularly perturbed systems with time delays. It is shown that if the boundary layer system is robustly globally asymptotically stable and if the reduced slow system admits a Lyapunov-Razumikhin function, then the singularly perturbed system has certain "practical and semi-global" asymptotic stability property as the parameter of singular perturbation reduces to zero.
\end{abstract}

\section{INTRODUCTION}

Singularly perturbed systems arise naturally in applications where a process evolves in different time scales. Such processes appear commonly in various fields ranging from physical and chemical systems to systems biology, see for instance, [1], [6], [12], [15] and references therein. Due to their wide applications, stability analysis for such systems has received much attention in a context of control theory, starting with the early work [5], see also [4]. Some more recent results were obtained in [1], [12], [15], [16] to deal with complex structures due to disturbances, unknown parameters, multi-stability, and modular behavior. However, much of the past work was on systems not affected by time delays, and most work for systems with time delays either focused on linear systems, or was based on linear approaches such as linearizations and exponential stability, see, for instance [6], [7]. For singularly perturbed linear functional-differential systems, a spectrum analysis approach was used to study exponential stability in [2]. For nonlinear systems, a method based on a small-gain approach was proposed in [17].

In this work we consider robust stability for singularly perturbed systems affected by both disturbances and time delays. Motivated by the ideas in [1], we establish sufficient conditions for a system to have certain type of practical and semi-global stability property based on stability properties of the boundary layer system and the reduced slow subsystems (see Theorem 1 in Section III). For the special case when the system is delay-free, our work extended the main result in [1] by removing the uniform property from the asymptotic stability assumption for the boundary layer system. Our work is also related to [12], where a unified framework for inputto-state stability was established for delay free systems with two time scales. The work [12] dealt with a very general case that covers various classical two time-scale problems, including the type of singularly perturbed systems studied in this current work for the spacial case when delays are not presented. On the other hand, in [12], the stability assumption for the boundary layer system is of a uniform nature in the

\footnotetext{
* This work was supported partially by NSF grant DMS-0906918.

${ }^{1}$ The authors are with the Department of Mathematical Sciences, Florida Atlantic University, Boca Raton, FL 33431, USA. Email: \{yyang8, ywang\} @ fau.edu
}

sense that the stability estimate is in "one measure", while in this current work, we relaxed this stability condition in "one measure" to a stability condition in "two measures" (which can also be phrased as output stability, see [10] and [13]). In the recent work [17], stability analysis for systems with delays was carried out based on a small-gain condition on the interconnections of the fast and slow subsystems. Comparing to the results obtained in [17], the results in this note are more applicable since the conditions are based on the decoupled reduced slow- and fast-subsystems. As initial steps for nonlinear systems, we consider in this work systems that are affected by delays only on slow variables. The more general cases remain to be topics for future studies.

A commonly used tool for systems with time-delays is the Lyapunov-Krasovskii theory. However, constructing a Lyapunov-Krasovskii functional can be much harder than constructing a Lyapunov function in the delay-free case (which already can be a challenging task). In this work, we will develop our result based on a Lyapunov-Razumikhin method, which is related to the small-gain theory. The connection between the classic Lyapunov-Razumikhin method and the small-gain theory in the context of input-to-state stability was initially recognized in the work [11]. The advantage of the Lyapunov-Razumikhin method is that it allows one to convert analysis of a system with delays to robust analysis of a delay-free system.

This note is organized as follows. In Section II we briefly discuss some preliminary results in stability analysis for systems with delays. We present our main results in Section III, and the main ideas of proofs in Section IV. Though our main theorem is only stated for systems with a single discrete delay, our result can be routinely generalized to the case of multi-discrete delays.

Notations. Throughout this note, we use $|\cdot|$ to denote the Euclidean norm of vectors, and $\|\cdot\|_{I}$ to denote the $L_{\infty}$ norm of measurable functions on the interval $I$, and $\|\cdot\|$ means the $L_{\infty}$ norm on $[0, \infty)$. For $q=\left(q_{1}, \cdots, q_{k}\right)$, we let $\|q\|_{I}=\max _{1 \leq i \leq k}\left\{\left\|q_{i}\right\|_{I}\right\}$. A function $\alpha: \mathbb{R}_{\geq 0} \rightarrow \mathbb{R}_{\geq 0}$ is of class $\mathcal{K}$ if it is continuous, positive definite, and strictly increasing; and is of class $\mathcal{K}_{\infty}$ if it is also unbounded. A function $\beta: \mathbb{R}_{\geq 0} \times \mathbb{R}_{\geq 0} \rightarrow \mathbb{R}_{\geq 0}$ is said to be of class $\mathcal{K} \mathcal{L}$ if for each fixed $t \geq 0, \beta(\cdot, t)$ is of class $\mathcal{K}$, and for each fixed $s \geq 0, \beta(s, t)$ decreases to 0 as $t \rightarrow \infty$. For any $\mathcal{K}$-function $\kappa$, we say that $\kappa<$ id if $\kappa(s)<s$ for all $s>0$. For a function of the form $Q\left(w_{1}, w_{2}, \ldots w_{m}\right)$, we use $D_{i} Q\left(w_{1}, w_{2}, \ldots, w_{m}\right)$ to denote the partial derivative of $Q$ in the $i$ th variable $w_{i}$. 


\section{PRELIMINARIES}

In this section we briefly review some basic results in stability analysis. Consider a system with time delays as in the following:

$$
\begin{aligned}
& \dot{x}(t)=f(x(t), x(t-\theta), d(t)), \\
& x(t)=x^{o}(t), \quad t \in[-\theta, 0],
\end{aligned}
$$

where for each $t \in \mathbb{R}, x(t) \in \mathbb{R}^{n}$ and $d(t) \in \mathbb{R}^{m}$. The parameter $\theta>0$ denotes the delay constant of the system. Assume that $f: \mathbb{R}^{n} \times \mathbb{R}^{n} \times \mathbb{R}^{n_{z}} \times \rightarrow \mathbb{R}^{n_{x}}$ is locally Lipschitz. The function $d: \mathbb{R}_{\geq 0} \rightarrow \mathbb{R}^{m}$, assumed to be measurable and locally essentially bounded, represents external inputs or disturbances.

Solutions of (1) are absolutely continuous functions that satisfy the equation almost everywhere. Under the assumptions stated above, the usual regularity conditions such as existence, uniqueness, and maximum extensions of solutions hold (see [3] and [14]). More precisely, for each continuous function $x^{o}(\cdot)$ defined on $[-\theta, 0]$ and each $d$, a unique solution of (1) satisfying the initial condition $x=x^{o}$ is defined on a maximum interval $[0, T)$ for some $T>0$.

A system as in (1) is said to be input-to-state stable (ISS) if there exists some $\beta \in \mathcal{K} \mathcal{L}$ and $\gamma \in \mathcal{K}$ such that

$$
|x(t)| \leq \max \left\{\beta\left(\left\|x^{o}\right\|_{[-\theta, 0]}, t\right), \gamma(\|d\|)\right\} .
$$

For a system without delay, the ISS property is defined as in (2) with $\|x\|_{[-\theta, 0]}$ replaced by $|x(0)|$. Various equivalent characterizations of the ISS property are known for systems without delays, in particular the Lyapunov description, see for instance [8] and [9]. For system with delays, there are two commonly used approaches: Lyapunov-Krasovskii method and Lyapunov-Razumikin method. In this work, we will follow the Lyapunov-Razumikin method, as discussed below.

For a system as in (1), we treat $x(t-\theta)$ as disturbances to the system, denoted by $w$, as in the following:

$$
\dot{x}(t)=f(x(t), w(t), d(t)) .
$$

The following was shown in [14]:

Lemma 2.1: Consider a system as in (1). Assume that (3) corresponding to (1) satisfies an ISs estimate with $(w, d)$ as input:

$$
|x(t)| \leq \max \{\beta(|x(0)|, t), \kappa(\|w\|), \gamma(\|d\|)\}
$$

for all $t \geq 0$. If $\kappa<\mathbf{i d}$, then (1) is ISS.

The next result was rooted in the Razumikhin theorem, whose connection to the ISS theory was established in [11]:

Lemma 2.2: Suppose that a system as in (1) admits a $C^{1}$ Lyapunov function $\mathbb{R}^{n} \rightarrow \mathbb{R}_{\geq 0}$ such that the following holds:

- for some $\underline{\alpha}, \bar{\alpha} \in \mathcal{K}_{\infty}$, it holds that

$$
\underline{\alpha}(|x|) \leq V(x) \leq \bar{\alpha}(|x|) \quad \forall x ;
$$

- for some $\kappa, \gamma \in \mathcal{K}_{\infty}$, it holds that

$$
\begin{aligned}
V(x) & \geq \max \{\kappa(V(w)), \gamma(|d|)\} \\
& \Rightarrow D V(x) f(x, w, d) \leq-\alpha(V(x)) .
\end{aligned}
$$

If $\kappa<\mathbf{i d}$, then (1) is ISS.

We will use frequently the following intermediate result between Lemma 2.1 and Lemma 2.2: Assume that (4) holds for some $\kappa \in \mathcal{K}$, then there exists $\beta \in \mathcal{K} \mathcal{L}$ such that along every trajectory of (1),

$|V(x(t))| \leq \max \left\{\beta(V(x(0)), t), \kappa\left(\|V(x)\|_{[-\theta, t]}\right), \gamma(\|d\|\}\right.$

on the maximum interval of the trajectory.

Below we consider a delay-free system:

$$
\dot{x}(t)=f(x(t), p),
$$

where $p$ is a time invariant parameter, taking values in $\mathbb{R}^{m}$, and the map $f: \mathbb{R}^{n} \times \mathbb{R}^{m}$ is locally Lipschitz.

Definition 2.3: A system as in (5) is said to be robustly globally asymptotically stable (robustly GAS) if there exists some $\beta \in \mathcal{K} \mathcal{L}$ such that

$$
|x(t)| \leq \beta(|(x(0), p)|, t)
$$

for all $t \geq 0$ and all $p \in P$.

For a give system as in (5), associate with it the following system:

$$
\dot{x}(t)=f(x(t), p(t)), \quad \dot{p}(t)=0,
$$

with an output map $y=h(x, p)=x$. Observe that the system (5) is robustly GAS if and only if the system (7) (with the state variable $\tilde{x}:=(x, p))$ is robustly output stable in the following sense:

$$
|y(t)| \leq \beta(|\tilde{x}(0)|, t) \quad \forall t \geq 0 .
$$

The following result can be derived from the main results in [13] (see also [10]):

Lemma 2.4: A system as in (5) is robustly GAS if and only if there exists a smooth Lyapunov function $V: \mathbb{R}^{n} \times \mathbb{R}^{m} \rightarrow$ $\mathbb{R}_{\geq 0}$ that satisfies the following:

- for some $\underline{\alpha}, \bar{\alpha} \in \mathcal{K}_{\infty}$,

$$
\underline{\alpha}(|x|) \leq V(x, p) \leq \bar{\alpha}(|(x, p)|)
$$

for all $x$; and

- there exists some $\alpha \in \mathcal{K}_{\infty}$ and some $\sigma \in \mathcal{K}$ such that

$$
D_{x} V(x, p) f(x, p) \leq-\alpha(V(x, p))
$$

for all $(x, p)$.

In fact, it was shown in [13] that the function $\alpha$ can be chosen as $\alpha(s)=s$. We use the more general function $\alpha$ for convenience of applications.

\section{MAin Results}

Consider a singularly perturbed nonlinear system with a time delay as in the following:

$$
\begin{aligned}
\dot{x}(t) & =f(x(t), x(t-\theta), z(t), d(t), \varepsilon), \\
\varepsilon \dot{z}(t) & =g(x(t), x(t-\theta), z(t), d(t), \varepsilon),
\end{aligned}
$$

where for each $t \in \mathbb{R},(x(t), z(t)) \in \mathbb{R}^{n_{x}} \times \mathbb{R}^{n_{z}}, d(t) \in \mathbb{R}^{m}$. The small parameter $\varepsilon$ takes values in $[0, \bar{\varepsilon}]$, and $\theta$ denotes the delay constant of the system. We assume that $f: \mathbb{R}^{n_{x}} \times$ $\mathbb{R}^{n_{x}} \times \mathbb{R}^{n_{z}} \times \mathbb{R}^{m} \times[0, \bar{\varepsilon}] \rightarrow \mathbb{R}^{n_{x}}$ and $g: \mathbb{R}^{n_{x}} \times \mathbb{R}^{n_{x}} \times \mathbb{R}^{n_{z}} \times$ $\mathbb{R}^{m} \times[0, \bar{\varepsilon}] \rightarrow \mathbb{R}^{n_{z}}$ are locally Lipschitz. The function $d:$ 
$\mathbb{R}_{\geq 0} \rightarrow \mathbb{R}^{m}$, assumed to be absolutely continuous, represents external inputs or disturbances.

Under the above assumption, the regularity properties such as existence, uniqueness, and maximum extension holds for solutions of (8). More precisely, for each continuous $\left(x^{o}, z^{o}\right)$ defined on $[-\theta, 0]$ and each input $d$, there is a unique solution $(x, z)$, satisfying $(x(t), z(t))=\left(x^{o}(t), z^{o}(t)\right)$ on $[-\theta, 0]$, defined on a maximum interval.

Assumption I. The algebraic system

$$
g\left(\sigma_{1}, \sigma_{2}, \sigma_{3}, d, 0\right)=0
$$

has a solution $\sigma_{3}=h\left(\sigma_{1}, \sigma_{2}, d\right)$, where $h: \mathbb{R}^{n_{x}} \times \mathbb{R}^{n_{x}} \times$ $\mathbb{R}^{m} \rightarrow \mathbb{R}^{n_{z}}$ is a $C^{2}$ (i.e., twice continuously differentiable) function.

Under Assumption I, when $\varepsilon=0$, system (8) reduces to

$$
\begin{aligned}
& \dot{x}(t)=f(x(t), x(t-\theta), z(t), d(t), 0), \\
& z(t)=h(x(t), x(t-\theta), d(t)) .
\end{aligned}
$$

Let $y(t)=z(t)-h(x(t), x(t-\theta), d(t))$, and consider the dynamics of the system in terms of the "fast time scale" $\tau$, where

$$
\tau=\frac{t}{\varepsilon}
$$

Let $\varphi(\tau)=x(\varepsilon \tau), \psi(\tau)=y(\varepsilon \tau)$. The $x$-subsystem of (8) becomes

$$
\frac{d}{d t} x(t)=F(x(t), x(t-\theta), y(t), d(t), \varepsilon),
$$

or equivalently,

$$
\frac{d}{d \tau} \varphi(\tau)=\varepsilon F\left(\varphi(\tau), \varphi_{\theta_{\varepsilon}}(\tau), \psi(\tau), d(\tau), \varepsilon\right)
$$

and the $z$-subsystem of (8) is transferred to

$$
\begin{aligned}
\frac{d}{d \tau} \psi(\tau) & =G\left(\varphi(\tau), \varphi_{\theta_{\varepsilon}}(\tau), \psi(\tau), \lambda_{1}(\tau), \varepsilon\right) \\
- & \varepsilon q\left(\varphi(\tau), \varphi_{\theta_{\varepsilon}}(\tau), \varphi_{2 \theta_{\varepsilon}}(\tau), \psi(\tau), \psi_{\theta_{\varepsilon}}(\tau), \lambda(\tau), \varepsilon\right),
\end{aligned}
$$

for all $\tau \geq \theta_{\varepsilon}$, where $\theta_{\varepsilon}=\theta / \varepsilon, \varphi_{s}(\tau)=\varphi(\tau-s)$,

$$
\begin{gathered}
\lambda(\tau)=\left(\lambda_{1}(\tau), \lambda_{2}(\tau), \lambda_{3}(\tau)\right) \\
=(d(\varepsilon \tau), d(\varepsilon \tau-\theta), \dot{d}(\varepsilon \tau)), \\
F\left(\mu_{0}, \mu_{1}, \nu_{0}, \lambda_{1}, \varepsilon\right)=f\left(\mu_{0}, \mu_{1}, \nu_{0}+h\left(\mu_{0}, \mu_{1}, \lambda_{1}\right), \lambda_{1}, \varepsilon\right), \\
G\left(\mu_{0}, \mu_{1}, \nu_{0}, \lambda_{1}, \varepsilon\right)=g\left(\mu_{0}, \mu_{1}, \nu_{0}+h\left(\mu_{0}, \mu_{1}, \lambda_{1}\right), \lambda_{1}, \varepsilon\right),
\end{gathered}
$$

and

$$
\begin{aligned}
& q\left(\mu_{0}, \mu_{1}, \mu_{2}, \nu_{0}, \nu_{1}, \lambda, \varepsilon\right)=D_{1} h F\left(\mu_{0}, \mu_{1}, \nu_{0}, \lambda_{1}, \varepsilon\right) \\
& \quad+D_{2} h F\left(\mu_{1}, \mu_{2}, \nu_{1}, \lambda_{2}, \varepsilon\right)+D_{3} h\left(\mu_{0}, \mu_{1}, \lambda_{1}\right) \lambda_{3} .
\end{aligned}
$$

Note that (12) is only valid for $\tau \geq \theta_{\varepsilon}$. On $[0, \theta]$, the $\psi$-subsystem becomes

$$
\begin{aligned}
& \frac{d}{d \tau} \psi(\tau)=G\left(\varphi(\tau), \varphi\left(\tau-\theta_{\varepsilon}\right), \psi(\tau), \lambda_{1}(\tau), \varepsilon\right) \\
& \quad-\varepsilon \hat{q}\left(\varphi(\tau), \varphi\left(\tau-\theta_{\varepsilon}\right), \psi(\tau), \psi\left(\tau-\theta_{\varepsilon}\right), \lambda_{1}(\tau), \lambda_{3}(\tau), \varepsilon\right) \\
& \quad-\varepsilon \zeta(\tau) \tilde{q}\left(\varphi(\tau), \varphi\left(\tau-\theta_{\varepsilon}\right), \lambda_{1}(\tau)\right),
\end{aligned}
$$

where $\zeta(\tau)=\dot{x}(\varepsilon \tau-\theta), \hat{q}\left(\mu_{0}, \mu_{1}, \mu_{2}, v_{0}, v_{2}, \lambda_{1}, \lambda_{3}, \varepsilon\right)$ is given by

$D_{1} h\left(\mu_{0}, \mu_{1}, \lambda_{1}\right) F\left(\mu_{0}, \mu_{1}, \psi, \lambda_{1}, \varepsilon\right)+D_{3} h\left(\mu_{0}, \mu_{1}, \lambda_{1}\right) \lambda_{3}$, and $\tilde{q}\left(\mu_{0}, \mu_{1}, \lambda_{1}\right)=D_{2} h\left(\mu_{0}, \mu_{1}, \lambda_{1}\right)$.

When $\varepsilon=0$ and when $y \equiv 0$, the $x$-subsystem (10) becomes the so called reduced slow system:

$$
\dot{x}(t)=F_{o}(x(t), x(t-\theta), d(t)),
$$

where $F_{o}\left(\mu_{0}, \mu_{1}, d\right)=F\left(\mu_{0}, \mu_{1}, 0, d, 0\right)$.

Assumption II. The reduced slow system (13) is ISS with $d$ as inputs and admits a $C^{1}$ Lyapunov-Razumikhin function $V$ that satisfies the following:

- for some $\underline{\alpha}^{x}, \bar{\alpha}^{x} \in \mathcal{K}_{\infty}$,

$$
\underline{\alpha}^{x}(|x|) \leq V(x) \leq \bar{\alpha}^{x}(|x|) \quad \forall x \in \mathbb{R}^{n} ;
$$

- for some $\kappa, \rho^{x} \in \mathcal{K}$, and some $\alpha^{x} \in \mathcal{K}^{\infty}$, it holds that

$$
\begin{aligned}
& V(x) \geq \max \left\{\kappa(V(w)), \rho^{x}(|d|)\right\} \\
& \quad \Rightarrow D V(x) F_{o}(x, w, d) \leq-\alpha^{x}(V(x)) ;
\end{aligned}
$$

- $\kappa(s)<s$ for all $s>0$.

When $\varepsilon=0$, the $\psi$-subsystem (12) system reduces to the following system (which is referred as boundary layer system):

$$
\frac{d}{d \tau} \psi(\tau)=G_{0}\left(\mu_{0}, \mu_{1}, \psi(\tau), d\right)
$$

where $\mu_{0}, \mu_{1}$ and $d$ are time invariant parameters, and $G_{0}\left(\mu_{0}, \mu_{1}, \psi, d\right)=G\left(\mu_{0}, \mu_{1}, \psi, d, 0\right)$.

Assumption III. The boundary layer system (15) with time invariant parameters $\left(\mu_{0}, \mu_{1}, d\right) \in \mathbb{R}^{n} \times \mathbb{R}^{n} \times \mathbb{R}^{m}$ is robustlyGAS.

By Lemma 2.4, Assumption III implies that (15) admits a smooth Lyapunov function $W: \mathbb{R}^{n_{x}} \times \mathbb{R}^{n_{x}} \times \mathbb{R}^{n_{z}} \times \mathbb{R}^{m} \rightarrow$ $\mathbb{R}_{\geq 0}$ satisfying the following:

- for some $\underline{\alpha}^{y}, \bar{\alpha}^{y} \in \mathcal{K}_{\infty}$,

$$
\underline{\alpha}^{y}(|\psi|) \leq W\left(\mu_{0}, \mu_{1}, \psi, \lambda_{1}\right) \leq \bar{\alpha}^{y}\left(\left|\left(\mu_{0}, \mu_{1}, \psi, \lambda\right)\right|\right),
$$

- there exists $\alpha^{y} \in \mathcal{K}_{\infty}$ such that

$$
D_{3} W G_{0}\left(\mu_{0}, \mu_{1}, \psi, \lambda_{1}\right) \leq-\alpha^{y}\left(W\left(\mu_{0}, \mu_{1}, \psi, \lambda_{1}\right)\right) .
$$

Below we state our main result:

Theorem 1: Consider a system as in (8) and suppose Assumptions I-III hold. Then there exist $\beta^{x}, \beta^{y} \in \mathcal{K} \mathcal{L}$, $\chi^{x}, \chi^{y} \in \mathcal{K}$ such that for each pair of positive numbers $(R, \delta)$, there is some $\varepsilon^{*}>0$ so that if

$$
\max \left\{\|x\|_{[-\theta, 0]},\|\dot{x}\|_{[-\theta, 0]},\|y\|_{[-\theta, 0]},\|d\|,\|\dot{d}\|\right\} \leq R,
$$

and if $\varepsilon \in\left[0, \varepsilon^{*}\right)$, it holds that

$$
\begin{aligned}
& |x(t)| \leq \max \left\{\beta^{x}\left(\|x\|_{[-\theta, 0]}+|y(0)|, t\right), \chi^{x}(\|d\|), \delta\right\}, \\
& |y(t)| \leq \max \left\{\beta^{y}\left(\|x\|_{[-\theta, 0]}+|y(0)|, \frac{t}{\varepsilon}\right), \chi^{y}(\|d\|), \delta\right\}
\end{aligned}
$$

for all $t \geq 0$. 
Remark 3.1: Note that the presence of $\|\dot{x}\|_{[-\theta, 0]}$ in the boundedness condition of the theorem is caused by the change of variables $y(t)=z(t)-h(x(t), x(t-\theta), d(t))$. The $y$-system is affected by values of $\dot{x}(t-\theta)$ when $t \in$ $[0, \theta]$. If the map $h\left(\sigma_{1}, \sigma_{2}, d\right)$ (as given in Assumption I) is independent of $\sigma_{2}$ (which happens, for instance, when the nominal $z$-subsystem is not affected by $x(t-\theta)$ ), the boundedness condition on $\|\dot{x}\|_{[-\theta, 0]}$ can be removed, and Theorem 1 will apply to all trajectories whose initial values are given by continuous functions.

Similarly, if the map $h\left(\sigma_{1}, \sigma_{2}, d\right)$ is independent of $d$, then the restriction on $\|\dot{d}\|$ can be removed, and Theorem 1 will apply to inputs that are measurable and locally essentially bounded.

\section{PRoOF OF THEOREM 1}

In this section, we sketch the main ideas and steps of the proofs of Theorem 1.

\section{A. The $\psi$-subsystem}

Consider the $\psi$-subsystem in (12). Let $W$ be as in (16)(17), and let $\hat{G}$ be a continuous function such that

$$
\begin{aligned}
& \left|G\left(\mu_{0}, \mu_{1}, \nu_{0}, \nu_{1}, \lambda, \varepsilon\right)-G_{0}\left(\mu_{0}, \mu_{1}, \nu_{0}, \nu_{1}, \lambda\right)\right| \\
& \leq \varepsilon \hat{G}\left(\mu_{0}, \mu_{1}, \nu_{0}, \nu_{1}, \lambda, \varepsilon\right)
\end{aligned}
$$

for all $\left(\mu_{0}, \mu_{1}, \nu_{0}, \nu_{1}, \lambda, \varepsilon\right)$. Along a trajectory of (10)-(12), one has

$$
\begin{aligned}
\frac{d}{d \tau} W\left(\varphi(\tau), \varphi\left(\tau-\theta_{\varepsilon}\right), \psi(\tau), \lambda_{1}(\tau)\right) \\
=D_{1} W \cdot \frac{d}{d \tau} \varphi(\tau)+D_{2} W \cdot \frac{d}{d \tau} \varphi\left(\varepsilon \tau-\theta_{\varepsilon}\right) \\
\quad+D_{3} W \cdot \frac{d}{d \tau} \psi(\tau)+D_{4} W \cdot \frac{d}{d \tau} d(\varepsilon \tau) \\
\leq-\alpha^{y}\left(W\left(\varphi(\tau), \varphi\left(\tau-\theta_{\varepsilon}\right), \psi(\tau), \lambda_{1}(\tau)\right)\right) \\
\quad+\varepsilon Q(\hat{\varphi}(\tau), \hat{\psi}(\tau), \lambda(\tau)), \varepsilon)
\end{aligned}
$$

for $\tau \geq \theta_{\varepsilon}$, where

$$
\begin{gathered}
\hat{\varphi}(\tau)=\left(\varphi(\tau), \varphi\left(\tau-\theta_{\varepsilon}\right), \varphi\left(\tau-2 \theta_{\varepsilon}\right)\right), \\
\hat{\psi}(\tau)=\left(\psi(\tau), \psi\left(t-\theta_{\varepsilon}\right)\right),
\end{gathered}
$$

and the function $Q(\hat{\mu}, \zeta, \hat{\nu}, \lambda, \varepsilon)$ is given by

$$
\begin{aligned}
& D_{1} W F\left(\mu_{0}, \mu_{1}, \nu_{0}, \lambda_{1}, \varepsilon\right)+D_{2} W F\left(\mu_{1}, \mu_{2}, \nu_{1}, \lambda_{2}, \varepsilon\right) \\
& -D_{3} W q\left(\mu_{0}, \mu_{1}, \mu_{3}, \nu_{0}, \nu_{1}, \lambda, \varepsilon\right)+D_{4} W \lambda_{3} \\
& +D_{3} W \hat{G}\left(\mu_{0}, \mu_{1}, \nu, \nu_{1}, \lambda, \varepsilon\right),
\end{aligned}
$$

with $\hat{\mu}=\left(\mu_{0}, \mu_{1}, \mu_{2}\right), \hat{\nu}=\left(\nu_{0}, \nu_{1}\right)$.

For $\tau \in\left[0, \theta_{\varepsilon}\right]$,

$$
\begin{aligned}
& \frac{d}{d \tau} W\left(\varphi(\tau), \varphi_{1}(\tau), \psi(\tau), \lambda_{1}(\tau)\right) \\
& \quad \leq-\alpha^{y}\left(W\left(\varphi(\tau), \varphi_{1}(\tau), \psi(\tau), \lambda_{1}(\tau)\right)\right) \\
& \left.\quad+\varepsilon Q_{0}\left(\hat{\varphi}(\tau), \dot{\varphi}\left(\tau-\theta_{\varepsilon}\right), \hat{\psi}(\tau), \lambda(\tau)\right), \varepsilon\right),
\end{aligned}
$$

where $Q_{0}(\hat{\mu}, \zeta, \hat{\nu}, \lambda, \varepsilon)$ is the function given by

$$
\begin{gathered}
D_{1} W F\left(\mu_{0}, \mu_{1}, \nu_{0}, \lambda_{1}, \varepsilon\right)+D_{2} W \cdot \zeta+D_{4} W \cdot \lambda_{3} \\
-D_{3} W \cdot(\hat{q}+\zeta \tilde{q})+D_{3} W \hat{G}\left(\mu_{0}, \mu_{1}, \nu, \nu_{1}, \lambda, \varepsilon\right) .
\end{gathered}
$$

For any $\delta>0$ given, let $\varepsilon_{\delta, R}>0$ be given so that

$$
\max \left\{\varepsilon\left|Q_{0}(\hat{\mu}, \zeta, \hat{\nu}, \lambda, \varepsilon)\right|, \varepsilon|Q(\hat{\mu}, \hat{\nu}, \lambda, \varepsilon)|\right\}<\delta
$$

for all $(\hat{\mu}, \zeta, \hat{\nu}, \lambda) \in D_{R}$, all $\varepsilon \in\left(0, \varepsilon_{\delta, R}\right)$, where $D_{R}$ is the set defined by where

$$
\begin{aligned}
\max \left\{V\left(\mu_{0}\right),\right. & V\left(\mu_{1}\right), V\left(\mu_{2}\right),|\zeta| \\
& \left.W\left(\mu_{0}, \mu_{1}, \nu_{0}, \lambda_{1}\right),|\lambda|\right\} \leq R .
\end{aligned}
$$

This is possible since $D_{R}$ is compact in the variables $\hat{\mu}, \zeta, \hat{\nu}$ and the functions $Q_{0}$ and $Q_{1}$ are continuous.

Let $(\varphi, \psi)$ be a trajectory of (11)-(12) for some $\varepsilon \leq \varepsilon_{\delta, R}$ and some $d$. Let $\xi(\tau)=V(\varphi(\tau))$ and

$$
\eta(\tau)=W\left(\varphi(\tau), \varphi\left(\tau-\theta_{\varepsilon}\right), \psi(\tau), \lambda_{1}(\tau)\right)
$$

Correspondingly, let $\mathcal{X}(t)=V(x(t))$, and

$$
\mathcal{Y}(t)=W(x(t), x(t-\theta), y(t), d(t))
$$

(so that $\xi(\tau)=\mathcal{X}(\varepsilon \tau)$ and $\eta(\tau)=\mathcal{Y}(\varepsilon \tau)$ ).

Assume that $\max \left\{\|V(x)\|_{[-\theta, 0]},\|\dot{x}\|_{[-\theta, 0]}\right\} \leq R$, and assume that for some $T>0$, it holds that

$$
\max _{0 \leq \tau<T}\{\xi(\tau), \eta(\tau),|\lambda(\tau)|\} \leq R
$$

then it holds on $[0, T)$ that

$$
\frac{d}{d \tau} \eta(\tau) \leq-\alpha^{y}(\eta(\tau))+\delta
$$

which in turn implies that on $[0, T)$,

$$
\eta(\tau) \leq \max \left\{\beta_{y}(\eta(0), \tau), \pi^{y}(\delta)\right\}
$$

where $\beta_{y} \in \mathcal{K} \mathcal{L}, \pi^{y} \in \mathcal{K}$.

Hence, we get the following result:

Lemma 4.1: Let $0<\delta<R$ and $\varepsilon \in\left(0, \varepsilon_{\delta, R}\right)$ be given. Consider a trajectory $(x(t), y(t))$ of (8). Assume that for some $T>0$, the following holds on $[0, T)$,

$$
\max _{0 \leq t<T}\left\{\mathcal{X}(t), \mathcal{Y}(t),\|\dot{x}\|_{[-\theta, 0]},|d(t)|,|\dot{d}(t)|\right\} \leq R
$$

Then

$$
\mathcal{Y}(t) \leq \max \left\{\beta_{W}(\mathcal{Y}(0), t / \varepsilon), \pi^{W}(\delta)\right\}
$$

for all $t \in[0, T)$.

Note that (21) leads to the following for $t \in[0, T)$ :

$$
|y(t)| \leq \max \left\{\beta_{y}(\mathcal{Y}(0), t / \varepsilon), \pi^{y}(\delta)\right\}
$$

where

$$
\beta_{y}(r, s)=\left(\underline{\alpha}^{y}\right)^{-1}\left(\beta_{W}(r, s)\right), \pi^{y}(r)=\left(\underline{\alpha}^{y}\right)^{-1}\left(\pi^{W}(r)\right) .
$$




\section{B. The $x$-subsystem}

To adopt the Razumikhin approach, we treat the $x$ subsystem (10) as

$$
\dot{x}(t)=F(x(t), w(t), y(t), d(t), \varepsilon),
$$

where $w$ is an input to the system. The delayed system (10) is resulted in under the feedback $w(t)=x(t-\theta)$.

Let $V$ be given as in Assumption II. It holds that

$$
\begin{aligned}
& D V(x) F(x, w, v, d, \varepsilon)=D V(x) F_{0}(x, w, d) \\
& \quad+D V(x)(F(x, w, v, d, \varepsilon)-F(x, w, 0, d, 0)) .
\end{aligned}
$$

It can be shown that there exist some $\mathcal{K}$-functions $\sigma, \gamma_{1}^{x}$, and $\rho^{x}$ and a continuous positive definite function $H$ such that

$$
\begin{aligned}
& |D V(x)(F(x, w, v, d, \varepsilon)-F(x, w, 0, d, 0))| \\
& \leq \max \left\{\sigma(V(x)) \cdot|v|, \sigma(V(w)) \cdot|v|, \gamma^{x}(|v|),\right. \\
& \left.\quad \rho_{1}^{x}(|d|), \varepsilon H(x, w, v, d)\right\}
\end{aligned}
$$

for all $x, w, v, d$ and $\varepsilon \in[0, \bar{\varepsilon}]$. It follows from (24) and Assumption II that

$$
\begin{gathered}
V(x) \geq \max \left\{\kappa(V(w)), \rho^{x}(|d|)\right\} \\
\Rightarrow \quad D V(x) F(x, w, v, d, \varepsilon) \leq-\alpha^{x}(V) \\
+\max \left\{\sigma(V(x))|v|, \sigma(V(w))|v|, \gamma^{x}(|v|),\right. \\
\left.\rho_{1}^{x}(|d|), \varepsilon H(x, w, v, d)\right\} .
\end{gathered}
$$

Reducing $\varepsilon_{\delta, R}$ if necessary, one may assume that for any $0<\delta<R$, it holds that $\varepsilon H(x, w, v, d)<\delta$ for all $\varepsilon \leq \varepsilon_{\delta, R}$, all $(x, w, v, d)$ such that

$$
\max \{V(x), V(w), W(x, w, v, d),|d|\} \leq R .
$$

One can then show that there exist some $\beta_{x} \in \mathcal{K} \mathcal{L}$ and $\mathcal{K}_{\infty}$-functions $\gamma_{1}^{x}, \gamma_{2}^{x}, \rho_{2}^{x}$, and $\pi^{x}$ such that for any given $0<\delta<R$ and $\varepsilon \in\left(0, \varepsilon_{\delta, R}\right)$, if a trajectory $(x(t), y(t))$ of (8) satisfies (20) for some $T>0$, then

$$
\begin{aligned}
V(x(t)) \leq \max \{ & \beta_{x}(V(x(0)), t), \kappa\left(\|V(x)\|_{[-\theta, t]}\right), \\
& \gamma_{1}^{x}\left(\|\sigma(V(x))\|_{[-\theta, t)} \cdot\|y\|_{[0, t]}\right), \\
& \left.\gamma_{2}^{x}\left(\|y\|_{[0, t]}\right), \rho_{2}^{x}(\|d\|), \pi^{x}(\delta)\right\}
\end{aligned}
$$

for all $t \in[0, T)$.

\section{Boundedness of trajectories}

For a given pair $(\delta, R)$, let

$$
\begin{aligned}
\Gamma(R)= & 2 \max \left\{\beta_{W}(R, 0), \beta_{x}(2 R, 0), \beta_{y}(R, 0)\right), \\
& \left.\gamma_{2}^{x}\left(\beta_{y}(R, 0)\right), \gamma_{1}^{x}\left(\sigma(2 R) \beta_{y}(R, 0)\right), \rho_{2}^{x}(R)\right\} .
\end{aligned}
$$

Choose $\delta_{1}>0$ such that

$$
\max \left\{\pi^{x}\left(\delta_{1}\right), \pi^{y}\left(\delta_{1}\right), \pi^{W}\left(\delta_{1}\right), \quad \gamma_{2}^{x}\left(\pi^{y}\left(\delta_{1}\right)\right)\right\} \leq \delta
$$

and

$$
\left.\gamma_{1}^{x}\left(\pi^{y}\left(\delta_{1}\right)\right) \cdot \sigma(s)\right) \leq \max \left\{\frac{s}{2}, \delta\right\}
$$

for all $s \in[0, \Gamma(R)]$. Since $\beta_{x}(R, 0) \geq R$, it follows that $\Gamma(R) \geq 2 R$. We also assume that $\delta \leq R$.

Consider a trajectory $(x(t), y(t))$ of (8). Let $\mathcal{X}, \mathcal{Y}$ be defined the same as in Section IV-A. Suppose the following holds:

$$
\begin{aligned}
\max \left\{\|\mathcal{X}\|_{[-\theta, 0]},\|\mathcal{Y}\|_{[-\theta, 0]},\right. \\
\left.\|\dot{x}\|_{[-\theta, 0]},\|d\|, \quad\|\dot{d}\|\right\} \leq R .
\end{aligned}
$$

It is not hard to show that that there exists some $t_{R}>0$ such that for all $\varepsilon \leq \varepsilon_{\delta, \Gamma(R)}$,

$$
V(x(t)) \leq 2 R \quad \forall t \in\left[0, t_{R}\right] .
$$

Let $\tilde{\varepsilon}_{R}>0$ be such that for all $\varepsilon \leq \tilde{\varepsilon}_{R}$

$$
\gamma_{1}^{x}\left(\sigma(\Gamma(R)) \cdot \beta_{y}\left(R, \frac{t_{R}}{\varepsilon}\right)\right) \leq R,
$$

and

$$
\gamma_{1}^{x}\left(\sigma(s) \cdot \beta_{y}\left(R, \frac{t_{R}}{\varepsilon}\right)\right) \leq \max \left\{\frac{s}{2}, \delta\right\}
$$

for all $0 \leq s \leq \Gamma(R)$.

Modifying $\varepsilon_{\delta, R}$ if necessary, we assume that $\varepsilon_{\delta_{1}, \Gamma(R)} \leq$ $\tilde{\varepsilon}_{R}$ for all $0<\delta<R$. The following can be proved:

Lemma 4.2: For $0<\delta<R$ given, let $\varepsilon \in\left(0, \varepsilon_{\delta_{1}, \Gamma(R)}\right)$. If a trajectory $(x(t), y(t))$ of (8) satisfies (29), then the trajectory is defined on $[0, \infty)$ and

$$
\max \{\mathcal{X}(t), \mathcal{Y}(t)\} \leq \Gamma(R)
$$

for all $t \geq 0$.

\section{The convergence property}

As a consequence of Lemma 4.2, if $\varepsilon<\varepsilon_{\delta_{1}, \Gamma(R)}$, and if (29) holds for a trajectory $(x(t), y(t))$, then the trajectory is defined on $[0, \infty)$, and (26) holds for $x$ and (21) holds for $y$ for all $t \geq 0$. Furthermore, one can refine estimate (26) as in the following.

Let $\varepsilon \in\left(0, \varepsilon_{\delta_{1}, \Gamma(R)}\right)$, and consider a trajectory $(x(t), y(t))$ for which (29) holds. Let

$$
\hat{R}=\max \left\{\|\mathcal{X}\|_{[-\theta, 0]}, \mathcal{Y}(0),\|d\|\right\},
$$

and let

$$
M_{\hat{R}}=\max \left\{\beta_{x}(2 \hat{R}, 0), \gamma_{2}^{x}\left(\beta_{y}(\hat{R}, 0)\right), \rho_{2}^{x}(\hat{R}),\right\} .
$$

Note that $\hat{R} \leq R$ and $M_{\hat{R}} \leq \Gamma(R)$. Then one can show the following fact:

$$
V(x(t)) \leq \max \left\{M_{\hat{R}}, \delta\right\} \quad \forall t \geq 0 .
$$

This implies that there exists some $\sigma_{0} \in \mathcal{K}_{\infty}$ such that

$$
\begin{gathered}
V(x(t)) \leq \max \left\{\sigma_{0}\left(\|x\|_{[-\theta, 0]}+|y(0)|\right),\right. \\
\left.\sigma_{0}(\|d\|), \delta\right\}
\end{gathered}
$$

for all $t \geq 0$.

To complete the proof of Theorem 1, let $0<\delta<R$ be given and let $\varepsilon \in\left(0, \varepsilon_{\delta_{1}, \Gamma(R)}\right)$. Let $(x(t), y(t))$ be a trajectory for which (29) holds. By Lemmas 4.1 and 4.2, the estimate 
(22) for $y$ holds for all $t \geq 0$. By (16), one concludes that there exists some $\hat{\beta}_{y} \in \mathcal{K} \mathcal{L}$ such that

$$
|y(t)| \leq \max \left\{\hat{\beta}_{y}\left(q\left(\|x\|_{[-\theta, 0]},|y(0)|,\|d\|\right), \frac{t}{\varepsilon}\right), \delta\right\}
$$

for all $t \geq 0$, where $q(r, s, t)=\max \{r, s, t\}$.

For the $x$-component, (26) implies that

$$
\begin{aligned}
V(x(t)) \leq & \max \left\{\beta_{x}(V(x(t / 2)), t / 2), \kappa\left(\|V\|_{[-\theta, t]}\right),\right. \\
& \gamma_{1}^{x}\left(\|\sigma(V)\|_{[(t / 2)-\theta, t]} \cdot\|y\|_{[t / 2, t]}\right), \\
& \left.\gamma_{2}^{x}\left(\|y\|_{[t / 2, t]}\right), \rho_{2}^{x}(\|d\|), \pi^{x}\left(\delta_{1}\right)\right\}
\end{aligned}
$$

for all $t \geq 0$. By (31),

$$
\begin{aligned}
\beta_{x}(V(x(t / 2)), t / 2) & \leq \max \left\{\sigma_{1}^{x}(\|d\|), \sigma_{2}^{x}(\delta),\right. \\
& \left.\hat{\beta}_{x}\left(\|x\|_{[-\theta, 0]}+|y(0)|, t\right)\right\}
\end{aligned}
$$

for all $t \geq 0$, where $\hat{\beta} \in \mathcal{K} \mathcal{L}, \sigma_{1}^{x} \in \mathcal{K}$, and $\sigma_{2}^{x} \in \mathcal{K}$. By (32), $\|y\|_{[t / 2, t]}$ is bounded by

$$
\max \left\{\hat{\beta}_{y}\left(q\left(\|x\|_{[-\theta, 0]},|y(0)|,|d(0)|\right), \frac{t}{2 \varepsilon}\right), \pi^{y}\left(\delta_{1}\right)\right\}
$$

for all $t \geq 0$.

One then can show that there exist some $\hat{\beta}_{x} \in \mathcal{K} \mathcal{L}, \hat{\rho}_{x} \in$ $\mathcal{K}$, and $\hat{\pi} \in \mathcal{K}$ such that

$$
\begin{aligned}
& \gamma_{1}^{x}\left(\|\sigma(V)\|_{[(t / 2)-\theta, t]} \cdot\|y\|_{[t / 2, t]}\right) \\
& \quad \leq \max \left\{\hat{\beta}_{x}\left(\|x\|_{[-\theta, 0]}+|y(0)|, t\right), \hat{\rho}_{x}(\|d\|), \hat{\pi}(\delta)\right\}
\end{aligned}
$$

for all $t \geq 0$. Combining with the decay estimate for $\|y\|_{[t / 2, t)}$, one sees that it follows from (33) that

$$
\begin{aligned}
V(x(t)) \leq \max \left\{\tilde{\beta}_{x}\left(\|x\|_{[-\theta, 0]}+|y(0)|, t\right),\right. \\
\left.\kappa\left(\|V\|_{[-\theta, t)}\right), \tilde{\rho}_{x}(\|d\|), \tilde{\pi}_{x}(\delta)\right\}
\end{aligned}
$$

for all $t \geq 0$, where $\tilde{\beta}_{x} \in \mathcal{K} \mathcal{L}, \tilde{\rho}_{x}, \tilde{\pi}_{x} \in \mathcal{K}$.

Hence, we have proved the following:

Lemma 4.3: For $0<\delta<R$ given, let $\varepsilon \in\left(0, \varepsilon_{\delta_{1}, \Gamma(R)}\right.$. If a trajectory $(x(t), y(t))$ satisfies (29), then (34) and (22) hold for all $t \geq 0$.

Finally, observe that (22) imlies that

$|y(t)| \leq \max \left\{\tilde{\beta}_{y}\left(\|x\|_{[-\theta, 0]}+|y(0)|, \frac{t}{\varepsilon}\right), \quad \tilde{\rho}_{y}(\|d\|), \tilde{\pi}_{y}(\delta)\right\}$

for all $t \geq 0$, where where $\tilde{\beta}_{y} \in \mathcal{K} \mathcal{L}, \tilde{\rho}_{y}, \tilde{\pi}_{y} \in \mathcal{K}$. In particular, by assuming $\varepsilon<1$, one has

$$
|y(t)| \leq \max \left\{\tilde{\beta}_{y}\left(\|x\|_{[-\theta, 0]}+|y(0)|, t\right), \tilde{\rho}_{y}\left(\|d\|, \tilde{\pi}_{y}(\delta)\right\}\right.
$$

for all $t \geq 0$. By Theorem 1 in [14], one concludes that there exist $\beta^{x}, \beta^{y} \in \mathcal{K} \mathcal{L}, \chi^{x}, \chi^{y} \in \mathcal{K}$, and $\pi \in \mathcal{K}$ such that

$$
|x(t)| \leq \max \left\{\beta^{x}\left(\|x\|_{[-\theta, 0]}+|y(0)|, t\right), \chi^{x}(\|d\|), \pi(\delta)\right\}
$$

for all $t \geq 0$.

\section{Conclusions}

We showed in this note that the ISS property of the reduced slow-system and the robust-GAsproperty of the boundary layer system hold robustly for a singularly perturbed system in the sense that for any given upper bounds on the magnitudes of the initial states, disturbances, and their derivatives; and a residual set of any given size, the trajectory will converge to the residual set if the perturbation parameter is small enough. It remains to be a future topic to relax the boundedness property on the derivatives of the functions that define the initial values of the trajectories.

\section{ACKNOWLEDGMENT}

The authors would like to thank an anonymous reviewer for valuable suggestions that led to improvements of the results.

\section{REFERENCES}

[1] P. D. Christofides AND A. R. TeEl, Singular perturbations and input-to-state stability, IEEE Transactions on Automatic Control, 41 (1996), pp. 1645-1650.

[2] V. Y. Glizer AND E. Fridman, Stability of singularly perturbed functional-differential systems: spectrum analysis and lmi approaches, IMA Journal of Mathematical Control and Information, 29 (2012), pp. 79-111.

[3] J. Hale And S. Lunel, Introduction to Functional Differential Equations, Springer-Verlag, 1993.

[4] H. K. KHaLIL, Nonlinear Systems, Prentice-Hall, Upper Saddle River, NJ, second ed., 1996.

[5] P. V. Koкotovic, H. Khalil, And J. O'Reilly, Singular Perturbation Methods in Control: Analysis and Design, Academic Press, London, 1986.

[6] X. LiU, X. SHEN, AND Y. ZHANG, Exponential stability of singularly perturbed systems with time delay, Applicable Analysis, 82 (2003), pp. 117-130.

[7] K. PARK AND J. LIM, Robust stability of non-standard nonlinear sigularly perturbed discrete systems with uncertainties, International Journal of Systems Science, iFirst (2012), pp. 1-9.

[8] E. SonTAG AND Y. WANG, On characterizations of the input-to-state stability property, Systems \& Control Letters, 24 (1995), pp. 351-359.

[9] E. Sontag AND Y. WANG, New characterizations of the input to state stability property, IEEE Transactions on Automatic Control, 41 (1996), pp. 1283-1294.

[10] E. SONTAG AND Y. WANG, Lyapunov characterizations of input to output stability, SIAM Journal on Control and Optimization, 39 (2001), pp. 226-249.

[11] A. TEEL, Connections between Razumikhin-type theorems and the ISS nonlinear small gain theorem, IEEE Transactions on Automatic Control, 43 (1998), pp. 960-964.

[12] A. TEEL, L. MOREAU, AND D. NEŠIĆ, A unified framework for inputto-state stability in systems with two time scales, IEEE Transactions on Automatic Control, 48 (2003), pp. 1526-1544.

[13] A. TEEL AND L. PRALY, A smooth converse Lyapunov function from a class- $\| \uparrow$ estimate involving two positive semidefinite functions, ESAIM: Control, Optimisation and Calculus of Variations, 5 (2000), pp. 313-315.

[14] S. TIWARI, Y. WANG, AND Z.-P. JIANG, Nonlinear small-gain theorems for large-scale time-delay systems, Dynamics of Continuous, Discrete and Impulsive Systems: Series A, 19 (2012), pp. 27-63.

[15] D. D. VeCCHIO AND J.-J. SLOTINE, A contraction theory approach to singularly perturbed systems with application to retroactivity attenuation, in Proceedings of the 50th IEEE Conference on Decision and Control and European Control Conference, 2011, pp. 5831-5836.

[16] L. WANG AND E. D. SonTAG, Singulaly perturbed monotone systems and an application to double phosphorylation cycles, Journal of Nonlinear Science, 18 (2008), pp. 527-550.

[17] Y. WANG AND Q. HUA, Stability analysis of nonlinear singularly perturbed systems with delays, Journal of Systems Science and Mathematical Sciences, 32 (2012), pp. 1239-1256. 